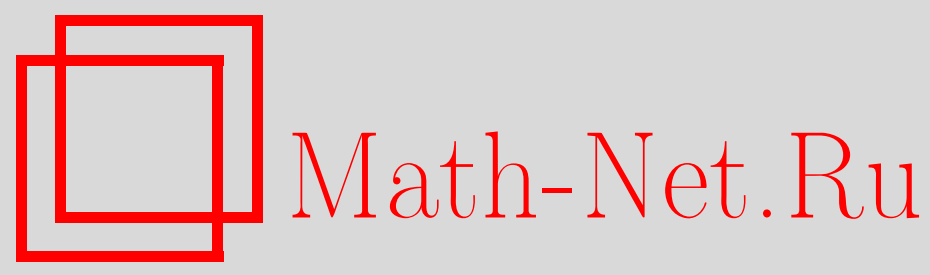

Б. И. Селиванов, Об одном классе статистик полиномиальных выборок, Дискрет. матем., 2009, том 21, выпуск 2, 126-137

DOI: https://doi.org/10.4213/dm1052

Использование Общероссийского математического портала Math-Net.Ru подразумевает, что вы прочитали и согласны с пользовательским соглашением http://www . mathnet.ru/rus/agreement

Параметры загрузки:

IP : 3.85 .73 .92

26 апреля 2023 г., 12:46:17 
УДК 519.2

\title{
Об одном классе статистик полиномиальных выборок
}

() 2009 г. Б. И. Селиванов

\begin{abstract}
Рассматриваются $M \geqslant 1$ независимых выборок, каждая из которых есть реализация некоторой полиномиальной схемы. Число исходов $N$ и число выборок $M$ фиксированы, объемы выборок неограниченно растут. Изучаются асимптотические свойства статистик вида $g(\bar{\xi})$, где $g(\bar{x})$ - дифференцируемая функция $M N$ вещественных переменных, $\bar{\xi}-$ вектор относительных частот исходов в выборках. Статистики такого рода играют важную роль в прикладном статистическом анализе.

В работе расширены возможности известного $\delta$-метода (метода линеаризации) в случае полиномиальных выборок. Доказана асимптотическая нормальность и сходимость по распределению статистик $g(\bar{\xi})$ к квадратичным формам от нормальных случайных величин (как для фиксированных вероятностей исходов в случае основной гипотезы, так и в случае контигуальных альтернатив к ней). Указаны условия обоих типов сходимости.

Работа выполнена при поддержке программой РАН «Современные проблемы теоретической математики» и гранта НШ 4129.2006.1 Совета по грантам при Президенте РФ для поддержки ведущих научных школ.
\end{abstract}

\section{1. Введение}

Рассмотрим $M \geqslant 1$ выборок $S_{1}, \ldots, S_{M}$, каждая из которых есть последовательность независимых испытаний с одним и тем же конечным множеством исходов $\{1, \ldots, N\}$. Если $M \geqslant 2$, то предполагаем, что выборки независимы друг от друга. Объемы выборок равны $n_{1}, \ldots, n_{M}$ соответственно. Будем использовать обозначения

$$
\bar{n}=\left(n_{1}, \ldots, n_{k}\right), \quad n=\sum_{k=1}^{M} n_{k}, \quad n_{0}=\min \left\{n_{1}, \ldots, n_{M}\right\} .
$$

Предположение, согласно которому вероятности появления исхода $i$ в каждом испытании выборки $S_{k}$ равны

$$
p_{i, k} \geqslant 0, \quad i=1, \ldots, N, \quad \sum_{i=1}^{N} p_{i, k}, \quad k=1, \ldots, M,
$$

назовем гипотезой $H$. Следовательно, в случае справедливости гипотезы $H k$-я из рассматриваемых выборок, $k=1, \ldots, M$, представляет собой реализацию полиномиальной 
схемы с вероятностями исходов, определяемыми вектором $\bar{p}_{k}=\left(p_{i, k}, \ldots, p_{N, k}\right)$. Введем обозначение

$$
\bar{p}=\left(\bar{p}_{1}, \ldots, \bar{p}_{M}\right)=\left(p_{1,1}, \ldots, p_{N, 1}, \ldots, p_{1, M}, \ldots, p_{N, M}\right) .
$$

Определим множество возможных альтернатив гипотезе $H$. Рассмотрим $M$ последовательностей полиномиальных схем

$$
\bar{\pi}_{k}=\left(\bar{p}_{k}(1), \ldots, \bar{p}_{k}\left(n_{k}\right)\right), \quad k=1, \ldots, M,
$$

где для $t=1,2, \ldots, n_{k}$ и $k=1, \ldots, M$

$$
\bar{p}_{k}(t)=\left(p_{1, k}(t), \ldots, p_{N, k}(t)\right), \quad p_{i, k}(t) \geqslant 0, \quad i=1, \ldots, N, \quad \sum_{i=1}^{N} p_{i}(t)=1,
$$

и компоненты вектора $\bar{p}_{k}(t)$ могут зависеть от $n_{k}$ (в этом случае имеет место схема серий). Будем говорить, что в выборке $S_{k}$ выполняется (простая) гипотеза $H\left(\bar{\pi}_{k}\right)$, если в ее $t$-м испытании реализуется полиномиальная схема, вероятности исходов которой задаются вектором (2). В случае, если выполняются равенства

$$
\bar{p}_{k}(1)=\bar{p}_{k}(2)=\ldots=\bar{p}_{k}\left(n_{k}\right)=\bar{p}_{k}, \quad \bar{p}_{k}=\left(p_{1, k}, \ldots, p_{N, k}\right), \quad k=1, \ldots, M,
$$

справедлива гипотеза $H$. В противном случае будем говорить об альтернативе основной гипотезе $H$, определяемой последовательностями $\bar{\pi}_{k}$. Эту альтернативу будем обозначать $H(\bar{\pi})$. В настоящей работе в качестве альтернатив гипотезе $H$ будут рассматриваться лишь те, которые контигуальны к $H$ в смысле Ле Кама [1] (см. (6)).

Пусть $v_{i, k}$ - частота и $\xi_{i, k}=v_{i, k} / n_{k}$ - относительная частота исхода $i$ в выборке $S_{k}$, $i=1, \ldots, N, k=1, \ldots, M$. Вектор, компонентами которого являются величины $\xi_{i, k}$, запишем в виде

$$
\bar{\xi}=\left(\xi_{1,1}, \ldots, \xi_{N, 1}, \ldots, \xi_{1, M}, \ldots, \xi_{N, M}\right) .
$$

В работе в предположении, что объемы всех выборок неограниченно растут (и, следовательно, $\left.n_{0} \rightarrow \infty\right)$, а $N$ и $M$ фиксированы, рассматриваются некоторые асимптотические свойства $M$-выборочных статистик вида $g(\bar{\xi})$, где $g(\bar{x})-$ вещественнозначная дифференцируемая функция $M N$ вещественных переменных, которая определена в $M N$-мерном вещественном единичном кубе

$$
K_{M, N}=\left\{\bar{x}: \bar{x}=\left(x_{1,1}, \ldots, x_{N, 1}, \ldots, x_{1, M}, \ldots, x_{N, M}\right)\right\}
$$

где $0 \leqslant x_{i, k} \leqslant 1, i=1, \ldots, N, k=1, \ldots, M$. Точки $\left(x_{1,1}, \ldots, x_{N, M}\right)$ куба $K_{M, N}$ будем обозначать $\bar{x}$. Класс указанных функций обозначим $C_{M, N}$.

Статистики такого рода играют важную роль в прикладном статистическом анализе. В частности, статистиками указанного вида в случае $M=1$ является известная статистика $\chi^{2}$ К. Пирсона (см. [2], §30.1) и статистика $\chi^{2}$, используемая для проверки независимости признаков в таблицах сопряженности признаков ([2], §30.5). В случае $M>1$ в этот класс (с некоторыми оговорками) можно включить статистику $\chi^{2}$, применяемую для проверки статистической однородности выборок. О статистике $\chi^{2}$ как критерии однородности см. [2], §30.6. В случае одной выборки к рассматриваемому классу статистик принадлежит семейство статистик, рассматриваемое в [3] и [4].

Цель настоящей работы - расширить возможности так называемого $\delta$-метода (см., например, [5], §6а.2), восходящего к Р. Мизесу, в случае статистик $g(\bar{\xi})$ полиномиальных 
выборок. Сообщение о предварительных результатах настоящей работы опубликовано в [6].

Работа состоит из трех параграфов. В параграфе 2 приведено несколько лемм, включая важную лемму 4, в которой указаны условия равенства нулю или положительности некоторой суммы. Эта лемма играет существенную роль при доказательстве четырех предельных теорем, приведенных в параграфе 3. Все эти теоремы доказываются с использованием $\delta$-метода. Теорема 1 , в которой идет речь об асимптотической нормальности статистик $g(\bar{\xi})$ в случае справедливости гипотезы $H$, является, по существу, простым примером использования $\delta$-метода. Доказательства теорем 2-4 иллюстрируют новые возможности $\delta$-метода применительно к статистикам полиномиальных выборок. В теореме 2 доказывается асимптотическая нормальность $g(\bar{\xi})$ в случае контигуальных альтернатив к гипотезе $H$. В теоремах 3 и 4 рассматривается сходимость по распределению статистик $g(\bar{\xi})$ к квадратичным формам от нормальных случайных величин. Эти случайные величины имеют нулевые средние значения, если выполняется гипотеза $H$ (теорема 3 ), а в общем случае ненулевые средние значения в случае близких альтернатив к гипотезе $H$ (теорема 4). Условия, при которых имеет место первый или второй тип сходимости статистик $g(\bar{\xi})$, находятся с помощью леммы 4 из параграфа 2.

\section{2. Вспомогательные результаты}

Лемма 1. Если верна гипотеза $H$, то случайный вектор

$$
\bar{\xi}_{0}=\left(\xi_{1,1}^{(0)}, \ldots, \xi_{N, 1}^{(0)}, \ldots, \xi_{M, 1}^{(0)}, \ldots, \xi_{N, M}^{(0)}\right)
$$

с компонентами

$$
\xi_{i, k}^{(0)}=\sqrt{n_{k}}\left(\xi_{i, k}-p_{i, k}\right), \quad i=1, \ldots, N, \quad k=1, \ldots, M,
$$

имеет нулевой вектор средних значений и ковариационную матрищу $\Sigma$ блочно-диагонального вида

$$
\Sigma=\operatorname{diag}\left(\Sigma_{1}, \ldots, \Sigma_{M}\right),
$$

гेe

$$
\Sigma_{k}=\left\|\sigma_{i, j}^{(k)}\right\|_{i, j=1}^{N}, \quad \sigma_{i, j}^{(k)}=p_{i, k}\left(\delta_{i, j}-p_{j, k}\right), \quad i, j=1, \ldots, N, \quad k=1, \ldots, M,
$$

$\delta_{i, j}-$ символ Кронекера, при $n_{0} \rightarrow \infty$ вектор $\bar{\xi}_{0}$ сходится по распределению к нормальному случайному вектору $\bar{\eta}=\left(\eta_{1,1}, \ldots, \eta_{N, 1}, \ldots, \eta_{1, M}, \ldots, \eta_{N, M}\right)$, который, как $u$ $\bar{\xi}_{0}$, имеет нулевой вектор средних значений и ковариационную матрииу $\Sigma$, определяемую формулами (4) и (5).

Утверждение леммы следует из независимости выборок $S_{1}, \ldots, S_{M}$ и многомерной центральной предельной теоремы с учетом известных фактов, связанных с полиномиальным распределением.

Лемма 2. Пусть верна альтернатива $Н(\bar{\pi})$, все вероятности (1) положительнь и выполняются условия

$$
\limsup _{n_{k} \rightarrow \infty} \sum_{t=1}^{n_{k}} \sum_{i=1}^{N}\left(p_{i, k}(t)-p_{i}\right)^{2} / p_{i}<\infty, \quad k=1, \ldots, M .
$$


Тогда случайный вектор

$$
\bar{\xi}_{1}=\left(\xi_{1,1}^{(1)}, \ldots, \xi_{N, 1}^{(1)}, \ldots, \xi_{1, M}^{(1)}, \ldots, \xi_{N, M}^{(1)}\right)
$$

с компонентами

$$
\xi_{i, k}^{(1)}=\left(1 / \sqrt{n_{k}}\right)\left(v_{i, k}-\sum_{i=1}^{n_{k}} p_{i, k}(t)\right), \quad i=1, \ldots, N, \quad k=1, \ldots, M,
$$

при $n_{0} \rightarrow \infty$ сходится по распределению к MN-мерному нормальному случайному вектору $\bar{\eta}$, определенному в формулировке леммы 1.

Доказательство леммы 2 аналогично доказательству теоремы 7.2 в [9] или леммы 3 в [10].

Замечание 1. Смысл условий (6) состоит в том, что в случае их выполнения альтернатива $H(\bar{\pi})$ будет контигуальна к $H$ (см. [9], §7).

Сформулируем также в виде отдельной леммы следующее простое утверждение. Положим

$$
\lambda_{i, k}\left(n_{k}\right)=\left(1 / \sqrt{n_{k}}\right) \sum_{t=1}^{n_{k}}\left(p_{i, k}(t)-p_{i, k}\right), \quad i=1, \ldots, N, \quad k=1, \ldots, M .
$$

Лемма 3. Для величин (8) в схеме серий выполняются соотношения

$$
\lim _{n_{k} \rightarrow \infty} \lambda_{i, k}\left(n_{k}\right)=\lambda_{i k}, \quad i=1, \ldots, N, \quad k=1, \ldots, M
$$

тогда и только тогда, когда вероятности $p_{i, k}(t)$ имеют вид

$$
p_{i, k}(t)=p_{i, k}+\frac{\lambda_{i, k}}{\sqrt{n_{k}}}+o\left(\frac{1}{\sqrt{n_{k}}}\right), t=1, \ldots, n_{k}, i=1, \ldots, N, k=1, \ldots, M,
$$

где $\lambda_{i, k}-$ константы, не зависящие от $n_{k}$.

Доказательство. Если в схеме серий существуют пределы (9), то при $n_{k} \rightarrow \infty$ для $k=1, \ldots, M$ выполняются равенства

$$
\sqrt{n_{k}}\left(p_{i, k}(t)-p_{i, k}\right)=\lambda_{i, k}+o(1), \quad i=1, \ldots, N,
$$

откуда следует (10). Верно и обратное.

Замечание 2. Вероятности (10) определяют так называемые гипотезы, близкие к $H$.

Докажем, наконец, следующее утверждение.

5 Дискретная математика, т.21 №2 
Лемма 4. Пусть

$$
a=\sum_{k=1}^{M} b_{k}\left(\sum_{i=1}^{N} p_{i, k} g_{i, k}^{2}-\left(\sum_{i=1}^{N} p_{i, k} g_{i, k}\right)^{2}\right)
$$

где $b_{k}$ - положительные числа, а $g_{i, k}$ - произвольные вещественные числа, $p_{i, k}$ вероятности (1), $i=1, \ldots, N, k=1, \ldots$, М. Пусть

$$
U_{k}=\left\{i: p_{i, k}>0, i=1, \ldots, N\right\}, \quad k=1, \ldots, M .
$$

Тогда выполняется неравенство $a \geqslant 0$, равенство $a=0$ имеет место тогда и только тогда, когда

$$
g_{i, k}=\gamma_{k}, \quad i \in U_{k}, \quad k=1, \ldots, M,
$$

$\gamma_{k}-$ константа, не зависящая от $i$.

Доказательство. Запишем формулу (11) в виде

$$
a=\sum_{k=1}^{M} b_{k} a_{k}
$$

где

$$
a_{k}=\sum_{i=1}^{N} p_{i, k} g_{i, k}^{2}-\left(\sum_{i=1}^{N} p_{i, k} g_{i, k}\right)^{2}, \quad k=1, \ldots, M
$$

Используя неравенство Коши-Буняковского, находим, что для $k=1, \ldots, M$

$$
\left(\sum_{i=1}^{N} p_{i, k} g_{i, k}\right)^{2}=\left(\sum_{i=1}^{N} \sqrt{p_{i, k}} \sqrt{p_{i, k}} g_{i, k}\right)^{2} \leqslant \sum_{i=1}^{N} p_{i, k} \sum_{i=1}^{N} p_{i, k} g_{i, k}^{2}=\sum_{i=1}^{N} p_{i, k} g_{i, k}^{2} .
$$

Это ввиду (1) доказывает неотрицательность величин (15). Теперь из (14) и положительности величин $b_{k}, k=1, \ldots, M$, следует неравенство $a \geqslant 0$.

Далее, рассмотрим $k$-ю, $k=1, \ldots, M$, из величин (15) и заметим, что согласно условию равенства в неравенстве Коши-Буняковского (см., например, [7], с. 80) соотношение $a=0$ выполняется тогда и только тогда, когда найдутся такие числа $\lambda$ и $\mu$, не зависящие от $i$ и не равные одновременно нулю, что

$$
\lambda g_{i, k} \sqrt{p_{i, k}}+\mu \sqrt{p_{i, k}}=0, \quad i=1, \ldots, N .
$$

Число $\lambda$ нулю равняться не может. В противном случае $\mu \neq 0$ и для рассматриваемого $k$ вероятности $p_{i, k}, i=1, \ldots, N$, равны нулю, что ввиду (1) невозможно. Отсюда вытекает, что условие (16) равносильно условию

$$
g_{i, k} \sqrt{p_{i, k}}=-\frac{\mu}{\lambda} \sqrt{p_{i, k}}, \quad i=1, \ldots, N,
$$

которое, в свою очередь, равносильно утверждению леммы. 


\section{3. Предельные теоремы для статистик $g(\bar{\xi})$}

Вспомнив обозначения, введенные в параграфе 1, положим

$$
\alpha_{k}(n)=n_{k} / n, \quad k=1, \ldots, M \text {. }
$$

В дальнейшем будем предполагать существование пределов

$$
\lim _{n_{0} \rightarrow \infty} \alpha_{k}(n)=\alpha_{k}>0, \quad k=1, \ldots, M, \quad \sum_{k=1}^{M} \alpha_{k}=1 .
$$

Следующее предложение служит отправной точкой наших дальнейших рассуждений.

Теорема 1. Если вероятности исходов в выборках $S_{1}, \ldots, S_{M}$ определяются гипотезой $H$, существуют предель $(18), g(\bar{x}) \in C_{M, N}$ и положительна величина

$$
\sigma=\sum_{k=1}^{M}\left(1 / \alpha_{k}\right)\left(\sum_{i=1}^{N} p_{i, k} g_{i, k}^{2}-\left(\sum_{i=1}^{N} p_{i, k} g_{i, k}\right)^{2}\right), \quad \sigma>0
$$

где

$$
g_{i, k}=\left.\frac{\partial}{\partial x_{i, k}} g(\bar{x})\right|_{\bar{x}=\bar{p}},
$$

то при $n_{0} \rightarrow \infty$ случайная величина

$$
\frac{\sqrt{n}}{\sqrt{\sigma}}(g(\bar{\xi})-g(\bar{p}))
$$

сходится по распределению к стандартной нормальной случайной величине, где векторь $\bar{\xi}$ и $\bar{p}$ определены в параграфе 1 .

Доказательство. Разложим функцию $g(\bar{x})$ в многомерный ряд Тейлора в точке $\bar{x}=\bar{p}$ и, как при доказательстве теоремы 6а.2 (II) в книге [5], с учетом обозначений (17) и леммы 1 получим равенство

$$
\sqrt{n}(g(\bar{\xi})-g(\bar{p}))=\sum_{k=1}^{M} \frac{1}{\sqrt{\alpha_{k}}(n)} \sum_{i=1}^{N} g_{i, k} \sqrt{n_{k}}\left(\xi_{i, k}-p_{i, k}\right)+o_{P}(1)
$$

где вектор $\bar{\xi}$ определен в параграфе $1, g_{i, k}-$ производные $(20)$ и $o_{P}(1)-$ случайная величина, которая при $n_{0} \rightarrow \infty$ сходится по вероятности к нулю. Согласно лемме 1 , сумма из правой части (22) является линейной комбинацией компонент асимптотически нормального случайного вектора $\bar{\xi}_{0}$ с компонентами (3). Отсюда с учетом (18) и вида ковариационной матрицы вектора $\bar{\xi}_{0}$, определяемой равенствами (4) и (5), следует, что эта сумма при $n_{0} \rightarrow \infty$ также асимптотически нормальна с параметрами $(0, \sigma)$. Для завершения доказательства теоремы 1 теперь достаточно воспользоваться предложением (Xb) в [5] на с. 118. 
Замечание 3. Если $M=1$, то ввиду асимптотической нормальности случайного вектора $\left(\sqrt{n_{1}}\left(\xi_{1,1}-p_{1,1}\right), \ldots, \sqrt{n_{1}}\left(\xi_{N, 1}-p_{N, 1}\right)\right)$ теорема 1 есть просто следствие леммы 1 и упоминавшейся теоремы 6а.2 (II) из [5]. В случае $M>1$ это уже не так. Однако теорему 6а.2 (II) можно без труда обобщить и сформулировать так, чтобы она была непосредственно применима к нескольким асимптотически нормальным векторным статистикам, относящимся к независимым выборкам.

Замечание 4. Для дисперсии суммы из правой части (22) ввиду (5) справедливо равенство

$$
\begin{aligned}
\mathbf{D}\left(\sum_{k=1}^{M}\left(1 / \sqrt{\alpha_{k}}(n)\right) \sum_{i=1}^{N} g_{i, k} \sqrt{n_{k}}\left(\xi_{i, k}-p_{i, k}\right)\right. \\
=\sum_{k=1}^{M}\left(1 / \sqrt{\alpha_{k}}(n)\right)\left(\sum_{i=1}^{N} g_{i, k}^{2} p_{i, k}\left(1-p_{i, k}\right)-\sum_{i, j=1, i \neq j}^{N} g_{i, k} g_{j, k} p_{i, k} p_{j, k}\right) .
\end{aligned}
$$

Отсюда и из (18) следует, что величина (19) есть предел при $n_{0} \rightarrow \infty$ дисперсии случайной величины, представляющей собой сумму из правой части (22). Для $M=1$ сумма (19) представляет собой частный случай выражения (6а.2.6) в [5] на с. 337 для произвольных асимптотически нормальных случайных величин. Условие равенства величины (19) нулю определяется леммой 4 и заключается в том, что производные (20) постоянны на множествах (12). Условие $\sigma=0$ в случае $M=1$ для статистики $\chi^{2}$ К. Пирсона исследовано автором в [8]. В общем случае подобное условие неизвестно.

Прежде, чем двигаться дальше, поясним смысл условия $n_{0} \rightarrow \infty$, которое присутствует во всех предельных теоремах, рассматриваемых в работе. Оно означает, что сходимость по распределению случайных величин, о которых идет речь, имеет место для произвольных последовательностей $\{\bar{n}\}$ таких, что $n_{0} \rightarrow \infty$. Напомним, что $\{\bar{n}\}$ есть вектор, компонентами которого являются объемы соответствующих выборок, а $n_{0}$ - минимальный из этих объемов.

Теперь рассмотрим асимптотическое поведение статистики (21) при альтернативах $H(\bar{\pi})$, контигуальных к гипотезе $H$.

Теорема 2. Пусть $g(\bar{x}) \in C_{M, N}$, все вероятности (1) положительны, для выборок $S_{1}, \ldots, S_{M}$ справедлива альтернатива $H(\bar{\pi})$ гипотезе $H$, определяемая последовательностями $\bar{\pi}_{k}$ векторов (2), причем для векторов (2) выполняются соотношения (6), сущчествует предел

$$
\lim _{n_{0} \rightarrow \infty} \sum_{k=1}^{M}\left(1 / \alpha_{k}\left(n_{k}\right)\right) \sum_{i=1}^{N} \lambda_{i, k}\left(n_{k}\right) g_{i, k}=m,
$$

где $g_{i, k}$ - производные (20) и величины $\lambda_{i, k}\left(n_{k}\right)$ определены равенствами (8), сумма (19) положительна и, наконеи, существуют предель (18).

Тогда при $n_{0} \rightarrow \infty$ случайная величина (21) асимптотически нормальна с параметрами $(m, 1)$.

Доказательство. Зафиксируем произвольную последовательность $\{\bar{n}\}$, для которой соответствующая последовательность $\left\{n_{0}\right\}$ стремится к бесконечности. Заметим, что это условие будет выполняться и для бесконечных подпоследовательностей $\{\bar{n}\}$. В дальнейшем указанное условие на $\{\bar{n}\}$ для краткости будем опускать. 
Компоненты (3) и (7) случайных векторов $\bar{\xi}_{0}$ и $\bar{\xi}_{1}$, соответственно, связаны равенствами

$$
\xi_{i, k}^{(0)}=\xi_{i, k}^{(1)}+\lambda_{i, k}\left(n_{k}\right), \quad i=1, \ldots, N, \quad k=1, \ldots, M
$$

Используя неравенство Коши-Буняковского и условие (6), находим, что существует константа $K>0$ такая, что для всех членов последовательности $\{\bar{n}\}$ выполняются неравенства $\lambda_{i, k}\left(n_{k}\right) \leqslant K, i=1, \ldots, N, k=1, \ldots, M$. Следовательно, из любой бесконечной последовательности $\left\{\bar{n}^{\prime}\right\}$, извлеченной из исходной последовательности $\{\bar{n}\}$, можно выбрать бесконечную подпоследовательность $\left\{\bar{n}^{\prime \prime}\right\}$, для которой существуют пределы

$$
\lim _{n_{0}^{\prime \prime} \rightarrow \infty} \lambda_{i, k}\left(n_{k}^{\prime \prime}\right)=\lambda_{i, k}<\infty, \quad i=1, \ldots, N, \quad k=1, \ldots, M,
$$

где $n_{k}^{\prime \prime}-k$-я компонента вектора $\bar{n}^{\prime \prime}$. При этом из $(18),(23)$ и (25) получаем равенство

$$
\sum_{k=1}^{M} \frac{1}{\alpha_{k}} \sum_{i=1}^{N} \lambda_{i, k} g_{i, k}=m
$$

Для каждого $\bar{n} \in\left\{\bar{n}^{\prime \prime}\right\}$ выполняется альтернатива $H(\bar{\pi})$ гипотезе $H$, определяемая последовательностями $\bar{\pi}_{k}$ векторов (2). Когда $\bar{n}$ пробегает подпоследовательность $\left\{\bar{n}^{\prime \prime}\right\}$ $\left(\right.$ при этом $\left.n_{0}^{\prime \prime} \rightarrow \infty\right)$, то, согласно лемме 2 , ввиду выполнения условий теоремы 2 и справедливости альтернативы $H(\bar{\pi})$, случайный вектор $\bar{\xi}_{1}$ с компонентами (7) сходится по распределению к $M N$-мерному нормальному случайному вектору с нулевым вектором средних значений и ковариационной матрицей (4). При этом, ввиду равенств (24), имеет место сходимость по распределению

$$
\bar{\xi}_{0} \Rightarrow \bar{\eta}_{0}=\left(\eta_{1,1}^{(0)}, \ldots, \eta_{N, 1}^{(0)}, \ldots, \eta_{1, M}^{(0)}, \ldots, \eta_{N, M}^{(0)}\right),
$$

где $\bar{\eta}_{0}-M N$-мерный нормальный случайный вектор с ковариационной матрицей (4) и средними значениями компонент

$$
\mathbf{E} \bar{\eta}_{i, k}^{(0)}=\lambda_{i, k}, \quad i=1, \ldots, N, \quad k=1, \ldots, M .
$$

Теперь, разлагая $g(\bar{x})$ в многомерный ряд Тейлора в точке $\bar{x}=\bar{p}$, получим, что

$$
g(\bar{x})-g(\bar{p})=\sum_{k=1}^{M} \sum_{i=1}^{N} g_{i, k}\left(x_{i, k}-p_{i, k}\right)+(1 / 2) \Theta\|\bar{x}-\bar{p}\|,
$$

где

$$
\|\bar{x}-\bar{p}\|=\sqrt{\sum_{k=1}^{M} \sum_{i=1}^{N}\left(x_{i, k}-p_{i, k}\right)^{2}}
$$

и $\Theta \rightarrow 0$, когда $\bar{x} \rightarrow \bar{p}$. Заменим в (29) переменные $x_{i, k}$ на соответствующие случайные величины $\xi_{i, k}$, определенные в параграфе 1 , и умножив обе части полученного равенства на $\sqrt{n^{\prime \prime}}$, с учетом (3), найдем, что

$$
\sqrt{n^{\prime \prime}}(g(\bar{\xi})-g(\bar{p}))=\sum_{k=1}^{M}\left(1 / \alpha_{k}\left(n^{\prime \prime}\right)\right) \sum_{i=1}^{N} g_{i, k} \xi_{i, k}^{(0)}+\frac{1}{2} \Theta^{*} \sqrt{\sum_{k=1}^{M}\left(n^{\prime \prime} / n_{k}^{\prime \prime}\right) \sum_{i=1}^{N}\left(\xi_{i, k}^{(0)}\right)^{2}} .
$$


С помощью стандартных рассуждений (см. [5], с. 336) можно убедиться, что при $n_{0}^{\prime \prime} \rightarrow \infty$ случайная величина $\Theta^{*}$ сходится по вероятности к нулю. Учитывая указанную выше асимптотическую нормальность случайного вектора $\bar{\xi}_{0}$ и существование пределов (18), приходим к выводу, что случайная величина

$$
\sqrt{\sum_{k=1}^{M}\left(n^{\prime \prime} / n_{k}^{\prime \prime}\right) \sum_{i=1}^{N}\left(\xi_{i, k}^{(0)}\right)^{2}}
$$

при $n_{0}^{\prime \prime} \rightarrow \infty$ имеет некоторое предельное распределение. Поэтому второе выражение в правой части (30) сходится по вероятности к нулю. Принимая во внимание этот факт и соотношения (18) и (27), получаем сходимость по распределению

$$
\sqrt{n^{\prime \prime}}(g(\bar{\xi})-g(\bar{p})) \Rightarrow \sum_{k=1}^{M}\left(1 / \alpha_{k}\right) \sum_{i=1}^{N} g_{i, k} \eta_{i, k}^{(0)} .
$$

Ввиду (26) и (28), математическое ожидание суммы из правой части (31) равно $m$, а дисперсия этой суммы есть $\sigma$, поскольку ковариационной матрицей вектора $\bar{\eta}_{0}$ является матрица (4).

Итак, когда $\bar{n}$ пробегает $\left\{\bar{n}^{\prime \prime}\right\}$, при $n_{0}^{\prime \prime} \rightarrow \infty$ случайная величина $\sqrt{n^{\prime \prime}}(g(\bar{\xi})-g(\bar{p})$ асимптотически нормальна с параметрами $(m, \sigma)$, и это верно для любой подпоследовательности $\left\{\bar{n}^{\prime \prime}\right\}$. Поэтому мы можем применить теорему о сходимости вероятностных мер (см., например, [11], с. 27) и, как в случае теоремы 1, используя предложение (Xb) из [5] на с. 118, завершить доказательство теоремы.

Замечание 5. Теорема 2 содержит в качестве частного случая асимптотическую нормальность статистики (21) в случае близких альтернатив, определяемых вероятностями вида (10).

Во второй части параграфа 3 будет рассмотрено асимптотическое поведение распределения $g(\bar{\xi})$ в случае, когда величина (19) равна нулю. Имеет место следующее утверждение.

Теорема 3. Пусть:

(1) функиия $g(\bar{x}) \in C_{M, N}$ имеет непрерывные частные производные второго порядка в окрестности точки $\bar{x}=\bar{p}$;

(2) вероятности исходов в каждой из выборок $S_{1}, \ldots, S_{M}$ определяются гипотезой $H$;

(3) сумма (19) равна нулю, $\sigma=0$;

(4) существуют предель (18).

Тогда при $n_{0} \rightarrow \infty$ статистика

$$
n(g(\bar{\xi})-g(\bar{p}))
$$

сходится по распределению к случайной величине

$$
\sum_{k, l=1}^{M} \sum_{i, j=1}^{N} \frac{1}{\sqrt{\alpha_{k} \alpha_{l}}} g_{i, k, j, l} \eta_{i, k} \eta_{j, l}
$$


- квадратичной форме от компонент нормального случайного вектора $\bar{\eta}$, который определен в формулировке леммы 1. Величины $g_{i, k, j, l}$ в (33) задаются равенством

$$
g_{i, k, j, l}=\left.\frac{1}{2} \frac{\partial^{2}}{\partial x_{i, k} \partial x_{j, l}} g(\bar{x})\right|_{\bar{x}=\bar{p}} .
$$

Доказательство. Воспользуемся формулой Тейлора для функций многих переменных и разложим функцию $g(\bar{x})$ в точке $\bar{x}=\bar{p}$, выписав, кроме линейных, члены второго порядка. Получим, что

$$
g(\bar{x})=g(\bar{p})+\sum_{k=1}^{M} \sum_{i=1}^{N} g_{i, k}\left(x_{i, k}-p_{i, k}\right)+\frac{1}{2} \sum_{k, l=1}^{M} \sum_{i, j=1}^{N} g_{i, k, j, l}\left(x_{i, k}-p_{i, k}\right)\left(x_{j, l}-p_{j, l}\right)
$$

где $\Theta_{1} \rightarrow 0$ при $\bar{x}=\bar{p}$ и величина $\|\bar{x}-\bar{p}\|$ имеет тот же смысл, что и в формуле (29). (Относительно используемой в настоящей работе формы остаточного члена в многомерном разложении Тейлора см. [12], §39). Далее, умножим обе части равенства (35) на $n$ и вместо переменных $x_{i, k}$ подставим в него случайные величины $\xi_{i, k}, i=1, \ldots, N, k=1, \ldots, M$. В результате, учитывая обозначения (3) и (17), получаем, что

$$
\begin{array}{r}
n(g(\bar{\xi})-g(\bar{p}))=n \sum_{k=1}^{M} \sum_{i=1}^{N} g_{i, k}\left(\xi_{i, k}-p_{i, k}\right)+\frac{1}{2} \sum_{k, l=1}^{M} \sum_{i, j=1}^{N} \frac{1}{\sqrt{\alpha_{k}(n) \alpha_{l}(n)}} g_{i, k, j, l} \xi_{i, k}^{(0)} \xi_{j, i}^{(0)} \\
+\Theta_{1}^{*}(\sqrt{n}\|\bar{\xi}-\bar{p}\|)^{2}
\end{array}
$$

Рассмотрим в правой части равенства (36) сумму

$$
\sum_{k=1}^{M} \sum_{i=1}^{N} g_{i, k}\left(\xi_{i, k}-p_{i, k}\right)
$$

Ввиду третьего предположения теоремы 3, величина $\sigma$, определяемая формулой (19), равна нулю. Отсюда и из леммы 4 следует, что для каждого $k=1, \ldots, M$ все величины $g_{i, k}$, соответствующие положительным $p_{i, k}, i=1, \ldots, N$, равны одному и тому же числу $\gamma_{k}$. Если же некоторое $p_{i, k}$ равно нулю, то с вероятностью 1 имеет место равенство $\xi_{i, k}=0$, и слагаемое $g_{i, k}\left(\xi_{i, k}-p_{i, k}\right)$ в сумме (37) также равно нулю. Поэтому для таких слагаемых, не боясь совершить ошибку, можно положить $g_{i, k}=\gamma_{k}$. Отсюда получаем, что

$$
\sum_{k=1}^{M} \sum_{i=1}^{N} g_{i, k}\left(\xi_{i, k}-p_{i, k}\right)=\sum_{k=1}^{M} \gamma_{k} \sum_{i=1}^{N}\left(\xi_{i, k}-p_{i, k}\right)=0
$$

и формула (36) с учетом определения нормы $\|\bar{\xi}-\bar{p}\|$ принимает вид

$$
n(g(\bar{\xi})-g(\bar{p}))=\frac{1}{2} \sum_{k, l=1}^{M} \sum_{i, j=1}^{N} \frac{1}{\sqrt{\alpha_{k}(n) \alpha_{l}(n)}} g_{i, k, j, l} \xi_{i, k}^{(0)} \xi_{j, l}^{(0)}+\Theta_{1}^{*}\left(\sum_{k=1}^{M} \sum_{i=1}^{N}\left(\xi_{i, k}^{(0)}\right)\right)^{2} .
$$

Заметим, что при $n_{0} \rightarrow \infty$ случайная величина $\Theta_{1}^{*}$ сходится по вероятности к нулю, а случайный вектор $\bar{\xi}_{0}$ по лемме 1 имеет предельное распределение (точнее, он асимптотически нормален). Поэтому второе выражение в (38) при $n_{0} \rightarrow \infty$ сходится по 
вероятности к нулю. Согласно лемме 1 и четвертому предположению теоремы, первое выражение в (38) при $n_{0} \rightarrow \infty$ сходится по распределению к случайной величине (33). Наконец, используя, как и при доказательстве теоремы 1, предложение (Xb) из [5] на с. 118, убеждаемся в справедливости теоремы 3.

В заключение сформулируем следующее обобщение теоремы 3, которое докажем, сочетая приемы, использованные ранее.

Теорема 4. Пусть выполнены первое, третье и четвертое условия теоремы 3, вероятности исходов в каждой из выборок $S_{k}, k=1, \ldots, M$, при $n_{k} \rightarrow \infty$ имеют вид (10), где числа $\lambda_{i, k}, i=1, \ldots, N$, - константыл, не зависящие от $n_{k} u p_{i, k}, i=1, \ldots, N$, $k=1, \ldots, M,-$ вероятности (1), которые предполагаются положительными.

Тогда при $n_{0} \rightarrow \infty$ статистика (32) сходится по распределению к квадратичной форме

$$
\sum_{k, l=1}^{M} \sum_{i, j=1}^{N} \frac{1}{\sqrt{\alpha_{k} \alpha_{l}}} g_{i, k, j, l} \eta_{i, k}^{(0)} \eta_{j, i}^{(0)}
$$

от компонент нормального случайного вектора $\bar{\eta}_{0}$, который определен в ходе доказательства теоремы 2 и имеет ковариационную матрииу (4) и средние значения компонент (28).

Доказательство. Как и при доказательстве теоремы 3, напишем разложение

$$
n(g(\bar{\xi})-g(\bar{p}))=Q_{1}+Q_{2}+Q_{3},
$$

где $Q_{1}, Q_{2}$ и $Q_{3}$ - первое, второе и третье выражения в правой части равенства (36). Первое из них $-Q_{1}-$ с вероятностью единица равно нулю. Последнее утверждение следует из того, что, ввиду предположения теоремы относительно вероятностей $p_{i, k}$, из леммы 4 вытекают равенства $g_{1, k}=\ldots=g_{N, k}, k=1, \ldots, M$. Далее, согласно (36),

$$
\begin{aligned}
Q_{2} & =\frac{1}{2} \sum_{k, l=1}^{M} \sum_{i, j=1}^{N} \frac{1}{\sqrt{\alpha_{k}(n) \alpha_{l}(n)}} g_{i, k, j, l} \xi_{i, k}^{(0)} \xi_{j, i}^{(0)}, \\
Q_{3} & =\Theta_{1}^{*} \sum_{k=1}^{M} \sum_{i=1}^{N}\left(\xi_{i, k}^{(0)}\right)^{2} .
\end{aligned}
$$

Нетрудно проверить, что если вероятности исходов в выборках $S_{1}, \ldots, S_{M}$ имеют вид (10), то выполняются условия (6). Воспользуемся леммой 2 и примем во внимание равенства (24), в которых, согласно лемме 3 , существуют пределы величин $\lambda_{i, k}\left(n_{k}\right)$. В результате мы придем к выводу, что при выполнении условий теоремы 4 случайный вектор $\bar{\xi}_{0}$ с компонентами $\xi_{i, k}^{(0)}$ сходится по распределению (см. (27)) к $M N$-мерному нормальному случайному вектору $\bar{\eta}_{0}$. Его ковариационная матрица определяется формулами (4) и (5), а средние значения компонент $\eta_{i, k}^{(0)}$ вектора $\bar{\eta}_{0}$ задаются равенствами (28). Наконец, воспользуемся векторной версией предложения (XII) и предложением (Xb) из [5] на c. 118-119 и увидим, что при $n_{0} \rightarrow \infty$ в сумме (39) слагаемое $Q_{3}$ сходится по вероятности к 0 , а слагаемое $Q_{2}$ сходится по распределению к квадратичной форме, указанной в формулировке теоремы. Это позволяет обычным путем завершить доказательство теоремы 4.

В заключение заметим, что задачи, связанные с распределением квадратичных форм от нормальных случайных величин, обсуждаются во многих работах (см., например, [13-16]), и здесь рассматриваться не будут. 


\section{Список литературы}

1. Гаек Я., Шидак 3., Теория ранговых критериев. Наука, Москва, 1971.

2. Крамер Г., Математические методы статистики. ГИИЛ, Москва, 1948.

3. Cressie N., Read T. R. C., Multinomial goodness-of-fit tests. J. Royal Statist. Soc. (1984) B46, 440-464.

4. Cressie N., Read T. R. C., Goodness-of-fit statistics for discrete multivariate data. Springer, Berlin, 1988.

5. Рао С. Р., Линейные статистические методы и их применения. Наука, Москва, 1968.

6. Селиванов Б. И., О статистиках-дифференцируемых функциях частот исходов $M$ полиномиальных выборок. Обозрение прикладной и промышленной математики (2005) 12, №4, 872-873.

7. Полиа Г., Сеге Г., Задачи и теоремы из анализа, 1. ГИТТЛ, Москва, 1956.

8. Селиванов Б. И., О предельных распределениях статистики $\chi^{2}$. Теория вероятностей и ее применения (1984) 29, №1, 132-134.

9. Селиванов Б. И., Об одном классе статистик типа хи-квадрат. Обозрение прикладной и промышленной математики (1995) 2, №6, 926-966.

10. Селиванов Б. И., Семейство многомерных статистик хи-квадрат. Дискретная математика (2002) 14, №3, 130-142.

11. Биллингсли П., Сходимость вероятностных мер. Наука, Москва, 1974.

12. Кудрявцев Л. Д., Курс математического анализа, 2. Высшая школа, Москва, 1981.

13. Chernoff H., Lehman E. L., The use of maximum likelihood estimates in $\chi^{2}$ tests for goodness of fit. Ann. Math. Statist. (1954) 25, №3, 579-586.

14. Good I. J., Condition for a quadratic form to have a chi-squared distribution. Biometrika (1969) 56, №1, 215-216.

15. Good I. J., Condition for a quadratic form to have a chi-squared distribution. Correction. Biometrika (1970) 57, №1, 225.

16. Dik J. J., Gunst M. C. M., The distribution of general quadratic form in normal variables. Statistica Neerlandica (14-26) 39, №1, 